\title{
Femtosecond dynamics of intramolecular charge transfer in 4-dimethylamino-4'-cyanostilbene in polar solvents
}

\author{
Nina Eilers-König ', Thomas Kühne, Dirk Schwarzer, \\ Peter Vöhringer ${ }^{2}$, Jörg Schroeder \\ Abteilung Spektroskopie und Photochemische Kinetik, Max-Planck-Institut für Biophysikalische Chemie, Am Faßberg. \\ D-37077 Göttingen, Germany
}

Received 4 December 1995

\begin{abstract}
The femtosecond dynamics of 4(N,N-dimethylamino)-4'-cyanostilbene (DCS) in acetonitrile and methanol solvent was studied by fluorescence upconversion and pump-probe absorption spectroscopy. The spectral evolution observed provides strong evidence for a fast internal charge-transfer (CT) process dominating the dynamics in the first few picoseconds, while Stokes shift dynamics seem to play only a minor role. The initially excited state seems to have already a large amount of CT character, in line with observations in jet-cooled DCS-solvent clusters. The non-exponential CT-state formation contains components significantly faster than longitudinal dielectric relaxation rates in the solvents studied, indicating a possible contribution by high-frequency vibrational modes.
\end{abstract}

\section{Introduction}

Intramolecular charge transfer (CT) in solution has attracted continued interest as it is a basic phenomenon underlying many chemical reactions that play a major role in, e.g., a variety of biological processes and laser dye dynamics [1-3]. Various theoretical models [4-6] have been proposed to describe excited-state intramolecular electron-transfer dynamics. The influence of the solvent environment

\footnotetext{
'Present address: Institut für Physikalische Chemie, Alexander-von-Humboldt Universität, Bunsenstr. 1, 10117 Berlin, Germany.

${ }^{2}$ Present address: Institut für Physikalische Chemie und Elektrochemie, Universität Karlsruhe, Kaiserstr. 12, 76128 Karlsruhe, Germany.
}

relaxation on electron-transfer rates has been investigated theoretically [2,4-6] and experimentally for intramolecular CT $[7,8]$ and bimolecular electron self-exchange reactions [9]. It appears that dynamical solvent effects can influence CT reactions if the transition from the initial 'locally' excited (LE) state to the CT state is adiabatic [5], i.e. if strong electronic coupling exists between the two states. In this case the LE $\rightarrow$ CT transition is assumed to proceed continually along the reaction coordinate on a common adiabatic potential energy surface. If $\mathrm{CT}$ takes place on a timescale comparable to or faster than that of the solvent dielectric relaxation, excited-state solvation dynamics may control the reaction $[4,5]$. In general, therefore, one may expect a complex evolution of time-resolved emission spectra reflecting both changes in electronic transition moments as a conse- 
quence of the LE $\rightarrow$ CT process as well as dynamic Stokes shifts caused by solvation effects.

There has been a number of studies on 4-(N,Ndimethylamino)-4'-cyanostilbene (DCS) in polar solution because it is suspected that immediately after photoexcitation DCS forms a TICT state. The dynamics of the presumed TICT state formation, however, has not yet been observed. The large Stokes shift of DCS in polar solvents was already investigated by Lippert et al. in 1959 [10]. Experiments to determine the dipole moment of trans-DCS were repeated later giving a value of $7 \mathrm{D}$ in the ground and $22 \mathrm{D}$ in the excited state [11]. Its emissive state was assumed to be of CT [12] or TICT character [13]. Recently time-resolved dual fluorescence in the excited state for high concentrations using high excitation intensities has been reported $[14,15]$ and ascribed to a dimer consisting of two excited DCS molecules (bicimer).

In our efforts to understand intramolecular substituent effects as well as the influence of the solvent environment on the photoisomerization dynamics of trans-stilbene [16-19], we recently investigated the dynamics in the $S_{1}$ state of jet-cooled isolated and solvent-complexed trans-DCS [20,21]. The results indicate that already a single water molecule is sufficient to stabilize the CT state to such an extent that it lies energetically only slightly above the LE state, which leads to significant coupling between these two states. Here we wish to extend these studies with femtosecond time resolution into the liquid phase, addressing the question whether there exists a precursor-successor relationship between the LE and CT state also in a polar liquid solvent environment.

\section{Experimental}

We performed pump-probe transient absorption and fluorescence upconversion measurements of solutions of trans-DCS in methanol and acetonitrile. We present only an overview of the experimental setup; details will be given elsewhere. The laser source consisted of a home-built regeneratively amplified titanium-sapphire laser which provided pulses of $200 \mathrm{fs}$ duration at a wavelength of $780 \mathrm{~nm}$. After amplification the pulse energy was $150 \mu \mathrm{J}$. Transmitting the laser beam through a KDP crystal yielded pulses with a pulse energy of $15 \mu \mathrm{J}$ at a wavelength of $390 \mathrm{~nm}$, which were used to excite the sample. For the fluorescence upconversion experiments excitation energies were attenuated to below $2 \mu \mathrm{J}$.

For the pump-probe absorption experiments a white-light continuum was generated by the remaining part of the laser beam in a sapphire crystal of 10 $\mathrm{mm}$ thickness. Selecting the wavelength with interference and coloured glass filters allowed us to record 20 absorption-time profiles between 450 and $700 \mathrm{~nm}$, which were then used to reconstruct transient pump-probe spectra using a pump-probe spectrum of DCS which was reported for a delay of 100 ps by Rettig et al. [15] as a reference for the infinite time limit.

The fluorescence upconversion setup was similar to the one described in Ref. [22]. The $780 \mathrm{~nm}$ pulses were used as gate pulses for the BBO upconversion crystal which had a thickness of $0.2 \mathrm{~mm}$. The resulting converted light was spectrally dispersed. It was possible to obtain 18 intensity-time profiles between 430 and $630 \mathrm{~nm}$. To reconstruct transient fluorescence spectra the integrated intensity-time profiles were adjusted to match steady-state fluorescence spectra of DCS measured by Zachariasse et al. [23]. After deconvolution the time resolution of both setups was well below $200 \mathrm{fs}$. All measurements were performed under magic-angle conditions for pump and probe beams.

Solvents used were of spectroscopic grade, DCS was prepared according to Ref. [24], and trans- and cis-isomers were separated by standard LC methods. The concentration of our samples was $3 \times 10^{-5}$ mol/1. Great care was taken to rule out the influence of the trans-cis isomerization of DCS on the time profiles. Samples of the pure cis-isomer were examined with the pump-probe setup. No transient absorption or gain was observed at the probe wavelengths used in the experiments.

\section{Results}

Recorded single-wavelength traces were fitted to a convolution of the Gaussian instrumental response function with a linear combination of at most three exponentials. The width of the instrumental response function and the point of time zero for each curve 

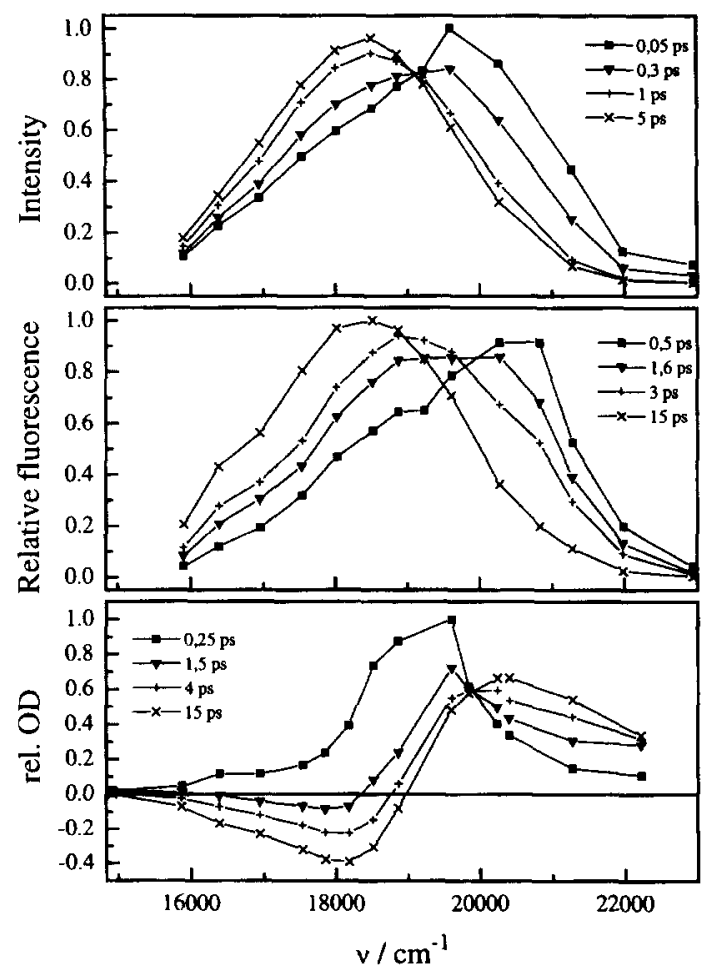

Fig. 1. Corrected fluorescence emission spectra of trans-DCS in acetonitrile (top) and methanol (center) and stimulated pumpprobe spectrum of trans-DCS in methanol at selected time delays between pump $(390-400 \mathrm{~nm})$ and gate $(780 \mathrm{~nm})$ or probe pulse. were treated as fitting parameters and allowed to vary within a reasonable range given by the independently measured pump-probe cross-correlation and linear frequency dispersion. The deconvoluted intensity-time profiles obtained in this way were then used to reconstruct the transient spectra at fixed time delays. To correct for the explicit frequency dependence of the emission and absorption cross section, spectral amplitudes were divided by $\nu$ for transient absorption and by $\nu^{4}$ for fluorescence spectra, $\nu$ being the frequency of the transition.

Reconstructed time-dependent fluorescence spectra of trans-DCS in methanol and acetonitrile and stimulated absorption/emission spectra in methanol are presented in Fig. 1. The fluorescence spectra in both solvents show the development of a red-shifted band from what has been a shoulder in the spectrum recorded immediately after excitation. In acetonitrile an isosbestic point at $\nu=19200 \mathrm{~cm}^{-1}$ is clearly visible, whereas in methanol the overlap of the two bands varies with time.

For further analysis the fluorescence spectra in Fig. 1 were fitted with log-normal functions as described by Maroncelli and Fleming [25]. A separation of the overlapping transient absorption and stimulated emission in methanol (spectra at the bottom of

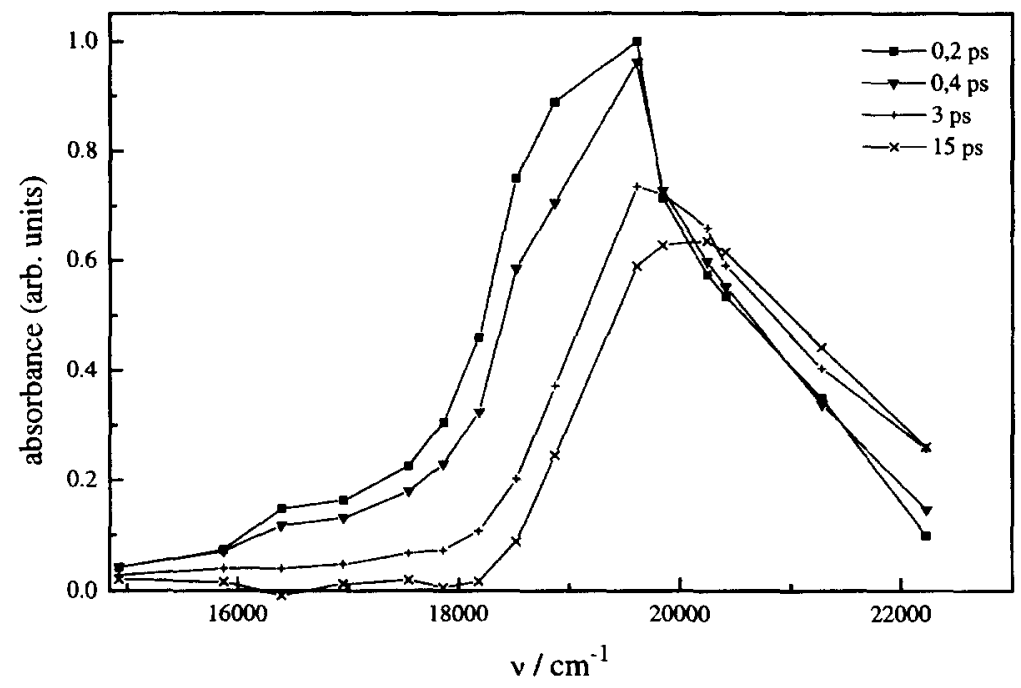

Fig. 2. Transient absorption spectra of DCS in methanol at selected time delays reconstructed from the pump-probe and emission spectra in Fig. 1. 
Fig. 1) was achieved under the assumption that at 15 ps time delay there is no residual transient absorption between 16000 and $18000 \mathrm{~cm}^{-1}$. The corresponding spectral region of the fluorescence spectrum was then amplitude scaled to match the corresponding stimulated emission spectrum, and subsequently subtracted from the pump-probe spectrum. The resulting transient absorption spectra of trans-DCS in methanol are shown in Fig. 2. They exhibit a temporary isosbestic point at $19800 \mathrm{~cm}^{-1}$ and a decay of the integrated spectral intensity on a picosecond timescale. A broad shoulder in the region of 16000 $18000 \mathrm{~cm}^{-1}$ disappears during the first two picoseconds. Log-normal functions were also fitted to the transient absorption bands.

From the fits to the spectra we calculated the time

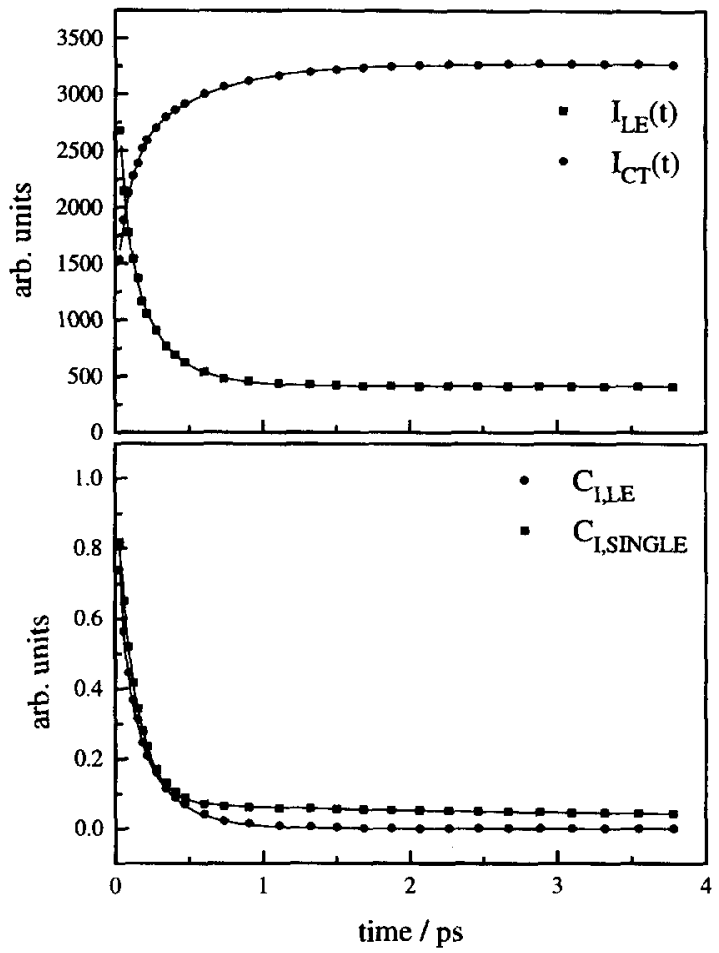

Fig. 3. Dynamics of trans-DCS fluorescence in acetonitrile. Top: time-dependence of integrated intensity of the two emission bands denoted as 'LE' and 'CT'. Points represent experimental data (not all shown), solid lines double exponential fit curves to the data. Bottom: intensity relaxation function of the 'LE'-band (O) (not all points shown) with double exponential fit (solid line), and the intensity relaxation function for the spectral band representation (E) together with a double exponential fit function (solid line).

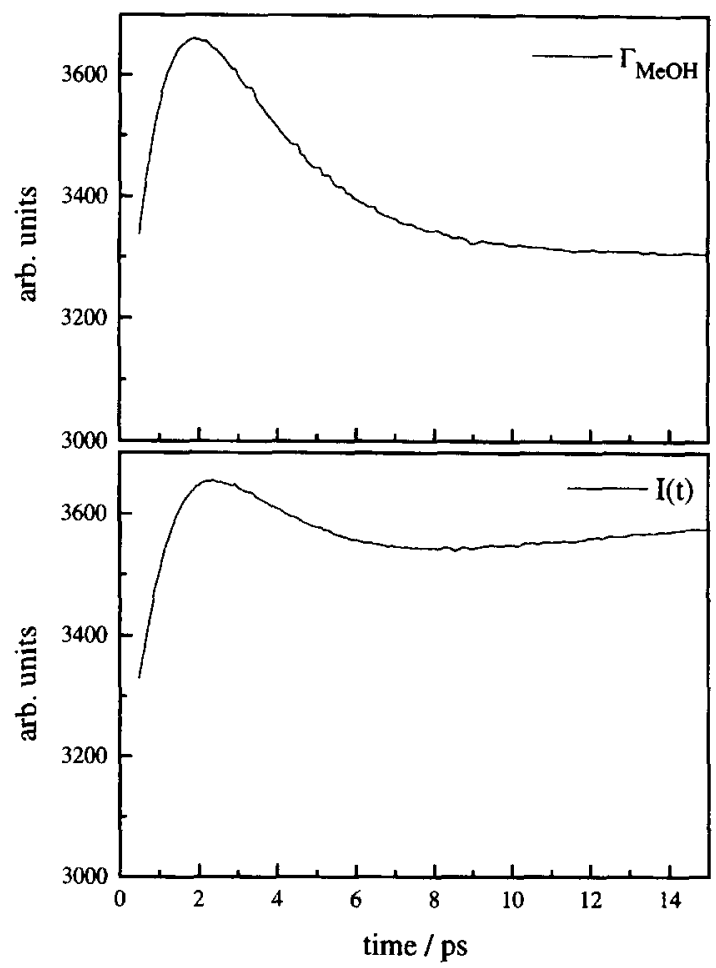

Fig. 4. Dynamics of trans-DCS fluorescence in methanol. Top: time-dependence of the width of the emission band (modelled by a single log-normal function). Bottom: time dependence of integrated emission intensity. (Not all experimental data points are shown.)

dependence of the integrated intensity $l(t)$, and - in the case of methanol - the spectral width $\Gamma(t)$. We also analyzed the data using an intensity relaxation function $C_{I}(t)$ analogously defined to the solvation energy relaxation function $C_{E}(t)$ for the time-dependent Stokes shift

$C_{I}(t)=\frac{I(t)-I(\infty)}{I(0)-I(\infty)}$.

Because of the distinct isosbestic point appearing in the time-resolved fluorescence spectra of trans-DCS in acetonitrile we used two log-normal fitting functions to represent the spectral evolution in this solvent. The resulting curves for the intensities of the two bands, denoted as LE (locally excited) and CT (charge transfer), are shown in Fig. 3, as well as the intensity relaxation function for the LE band. It can be seen that the rise of the CT band matches the decay of the LE band. 


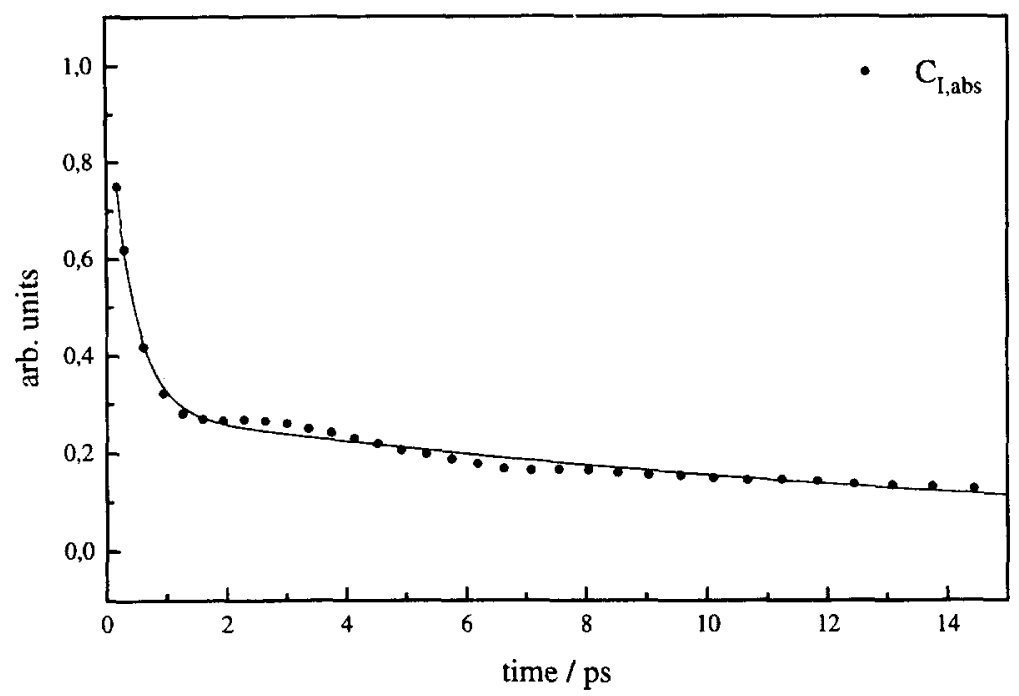

Fig. 5. Intensity relaxation function of the transient absorption of trans-DCS in methanol (not all experimental points shown) and double exponential fit (solid line).

In methanol, the spectra could not be resolved into two distinct bands. Therefore, we fitted them as a first step - with a single log-normal function. The curves for $\Gamma(t)$ and $I_{\mathrm{fl}}(t)$ for methanol in Fig. 4 exhibit a non-monotonous behaviour, which may indicate that the representation by a single band may not be sufficient. $\Gamma(t)$ exhibits a maximum of 3650 $\mathrm{cm}^{-1}$ at $t \approx 2 \mathrm{ps}$, followed by a slow decrease to $3200 \mathrm{~cm}^{-1}$. The integrated intensity $I_{\mathrm{fl}}(t)$ of the fluorescence band changes only by about $10 \%$. Its temporal behaviour up to $10 \mathrm{ps}$ mainly reflects the development of $\Gamma(t)$. In contrast, the integrated transient absorption intensity $I_{\mathrm{a}}(t)$ of trans-DCS in methanol is characterized by a non-exponential monotonous decay similar to the fluorescence intensity decay in acetonitrile. An error in the measured fluorescence spectrum in methanol due to uncertainties in the scaling and low signal intensities for frequencies above $21000 \mathrm{~cm}^{-1}$ would not seriously influence the shape and area of the calculated transient absorption band, as the respective frequencies are well off the maximum to the high-energy side, where transient absorption dominates the pump-probe measurements on the investigated timescale. If, at early times, the real fluorescence intensity was higher in this spectral region, the decrease in $I_{\mathrm{a}}(t)$ would be even more pronounced. Thus, the intensity relaxation function of the absorption band in methanol shown in Fig. 5 would not change significantly.

\section{Discussion}

Although the observation of dual fluorescence has already been reported for trans-DCS in solution $[14,15]$, this is the first time it has been observed on a femtosecond timescale for low concentrations of DCS using moderate excitation powers ( $<10 \mathrm{MW})$, meaning that the bicimer model [14] is not applicable to the interpretation of excited-state relaxation of trans-DCS for our experimental conditions. In contrast, the experiments presented here give us insight into the ultrafast dynamics of the trans-DCS monomer within the first few picoseconds of excitation. We first turn to a discussion of the results in acetonitrile.

The spectral time evolution of the fluorescence in this solvent shows a clear isosbestic point which we relate to a transition between two distinct, barrierseparated states after photoexcitation into the first excited singlet state $\left(S_{1},{ }^{\prime} L E '\right)$ of trans-DCS. Modelling the relaxation process, as discussed in the previous section, by two fluorescence bands with fixed first and second moments and variable amplitudes results in a non-exponential rise of the long- 
wavelength ('CT') band and a corresponding fall of the short-wavelength band with similar time constants. The time evolution of the integrated fluorescence (Fig. 3) can be fit by a sum of two exponentials with time constants $\tau_{\mathrm{LE} 1}=60 \mathrm{fs}$ and $\tau_{\mathrm{LE} 2}=250$ fs for the fall and $\tau_{\mathrm{CT} 1}=80 \mathrm{fs}$ and $\tau_{\mathrm{CT} 2}=510 \mathrm{fs}$ for the rise, respectively, with about $50 \%$ relative amplitude each. In view of the difficulties in precisely separating the two bands at early times, the agreement between the two sets of coefficients provides evidence for a precursor-successor relationship between the two states responsible for the electronic transition.

The relaxation process cannot be the photoisomerization of DCS on the $S_{1}$ potential energy surface, since we found that the cis-isomer of DCS does not fluoresce. The red-shifted emission is therefore assigned to an intramolecular CT state. A transition of the type LE $\rightarrow$ CT has been proposed before [12], but the experimental evidence was not sufficient to distinguish between the effects of a CT transition and a dynamic Stokes shift.

The close agreement of the fluorescence intensity relaxation function $C_{l, \mathrm{LE}}(t)$ of the LE band with the corresponding function $C_{l, \text { Single }}(t)$ obtained from fitting the spectral evolution with a single emission band as shown in Fig. 3 (bottom) indicates that the spectral dynamics within the first few hundred femtoseconds is completely dominated by the depletion of the LE state. This is proof that, also in this case, already the time-dependent total emission intensity $I_{\mathrm{fl}}(t)$ provides sufficient information regarding the CT dynamics [26]. The decrease in the total emission intensity by radiative transition to the ground state can be ruled out since the lifetimes of the fluorescent state at $25^{\circ} \mathrm{C}$ were found to be $480 \mathrm{ps}$ in methanol and $580 \mathrm{ps}$ in acetonitrile [23], respectively.

The larger time constant of the non-exponential LE depletion of $250 \mathrm{fs}$ is very close to the longitudinal dielectric relaxation time of acetonitrile of $200 \mathrm{fs}$ obtained from dielectric loss spectroscopy [27], suggesting that this 'slower' part of the intramolecular CT dynamics seems to be governed by solvent relaxation. This contribution would account for about half the LE population decaying due to an adiabatic CT process. The initial $50 \%$ of the population decay on a timescale four times faster, either indicating higher frequency contributions to the solvent response
$[28,29]$ that couple only to about one half of the solvated DCS molecules, or the influence of initially excited intramolecular vibrational modes promoting a $\mathrm{CT}$ process faster than solvent dynamics as envisaged in the extended Sumi-Marcus model [4,8]. For a meaningful discussion of the applicability of the corresponding models one needs to know (i) the spectral density function of the solvent as obtained from optical Kerr effect measurements [28,29], and (ii) the vibrational spectrum of the $S_{1}$ state of transDCS in acetonitrile as it could be obtained from femtosecond vibrational dephasing experiments. An analysis of additional experiments along these lines will be given in a future publication. The existence of differently solvated species in DCS-acetonitrile solutions seems to be highly probable in view of recent results on jet-cooled solvate clusters of DCS with acetonitrile [21].

In contrast to the observations in acetonitrile the time-dependent fluorescence spectra of trans-DCS in methanol do not exhibit an isosbestic point, although also two emission bands seem to be present. As the separation into two bands and their numerical representation is fairly ambiguous in this case, we represent the time evolution of the emission by the parameters of a single band. The FWHM of $\Gamma(t)$ for spectra in methanol in Fig. 4 exhibits a maximum, while the integrated intensity $I(t)$ of the total emission changes non-monotonously, but very little, mainly reflecting the corresponding behaviour of $\Gamma(t)$. This indicates that the description in terms of a single band is inadequate. Clearer signs of a CT process taking place like in acetonitrile, though, can be found in the evolution of the transient absorption spectra in Fig. 2, which shows an isosbestic point roughly up to $1.5 \mathrm{ps}$ after excitation. Also, the low-frequency shoulder of the transient absorption band disappears during the first $1.5 \mathrm{ps,}$, and the time dependence of the integrated absorption intensity was found to decrease in a way similar to the LE fluorescence in acetonitrile. This suggests that the spectral dynamics also in this solvent are probably dominated by intramolecular CT formation. One has to be aware of the complication, however, that possible changes in the electronic properties of the upper excited state reached in transient absorption might also contribute to some extent to the time dependence of the spectrum. 
The difference in spectral evolution of the DCS fluorescence between the two solvents may either reflect the more complex dielectric response of the hydrogen-bonded solvent methanol or be an indication of specific solvation effects in the system DCS-methanol. Dielectric relaxation in alcohols takes place on various largely different timescales. For methanol one obtains from dielectric loss spectroscopy values of $9.4,1.1$ and $0.10 \mathrm{ps}$ for the longitudinal relaxation times in a three-component model [27]. These values agree with the timescale of the transient absorption changes. It might be that the fast components induce charge separation, and that the slow component prevents an isosbestic point of the DCS absorption to be seen in methanol on the longer timescale, as it would cause a dynamical spectral shift of the CT state absorption. The effect would, of course, be much more pronounced for the emission band and might account for the failure of the attempt to separate the spectrum into two distinct bands.

On the other hand, investigations of small clusters of trans-DCS with methanol have shown that the fluorescence excitation spectra differ appreciably from those obtained with acetonitrile, and that, in particular, already in $1: 1$ complexes of trans-DCS and methanol there is evidence for a strong mixing between the locally excited state and the CT state [21]. This might suggest another probable reason for the differences between DCS fluorescence dynamics in methanol and acetonitrile, and concerns the different character of the initially excited state in the two solvents.

Solvent-induced electron transfer has been reported for bianthryl [8] and for DCM [29]. In both cases, a barrier along the reaction coordinate separating initially prepared and CT state compounds apparently ensured the applicability of electron-transfer models to explain the influence of dynamical solvent effects on the internal CT process. We think, however, that the initially prepared state of DCS, depending on the solvent environment, is more or less a mixture of LE and CT contributions. We estimated the first moment $\langle\nu(0)\rangle$ of the hypothetical time-zero emission spectrum of DCS to be $21300 \mathrm{~cm}^{-1}$ by subtracting the value of the Stokes shift in the nonpolar solvent n-pentane from the first spectral moment of the absorption band in acetonitrile. Although the early fluorescence spectra in the polar solvents show some structure reminescent of the stationary emission spectrum in n-pentane, they are spectrally displaced by $1600 \mathrm{~cm}^{-1}$ (methanol) and $2200 \mathrm{~cm}^{-1}$ (acetonitrile) to the red from the estimated time-zero spectrum. Under the assumption that the dipole moments for the ground state and the CT state are 7 and $22 \mathrm{D}$, respectively [11], the dipole moment of the state corresponding to the short-wavelength emission band in acetonitrile was calculated from the Stokes shift relative to $\langle\nu(0)\rangle$ using the Ooshika-LippertMataga equation $[10,31,32]$ to be as large as $20 \mathrm{D}$. Although this estimate has to be considered only as qualitative, it seems to suggest a strong CT contribution to the initially excited state.

Steady-state spectroscopy also strengthens the assumption of a highly polar, initially excited state. The stationary absorption and emission spectra of trans-DCS in numerous solvents show a dependence of the position of their maxima on solvent polarity [33]. As has been pointed out for 4-aminophthalimide [34], it can be concluded that the initially populated state has already strong CT character.

\section{Conclusion}

We have shown that the optically prepared initial state of trans-DCS in acetonitrile and methanol solution evolves on a timescale of about a picosecond into a successor state which we identify as the intramolecular CT state. The spectral dynamics of the emission in acetonitrile are completely determined by this CT process, and the longer timescale of the non-exponential decay of the intensity correlation function coincides with the longitudinal dielectric relaxation time of acetonitrile. The faster second component probably indicates contributions of $\mathrm{CT}$ channels from higher vibrationally excited states which are faster than solvent relaxation.

In methanol the picture is more complicated, although the transient absorption decay also seems to indicate a fast CT process dominating the dynamics at early times. In this solvent, however, an additional dynamic Stokes shift on a longer timescale in part overlaps with the internal CT process.

The initially prepared state in both solvents already seems to contain a strong admixture of CT 
character, in agreement with corresponding observations in jet-cooled DCS-solvent complexes [20,21].

\section{Acknowledgement}

The authors would like to thank Professor Dr. Jürgen Troe for many stimulating discussions and generous support. We also thank Dr. W. Kühnle for the preparation and purification of DCS. Financial support by the Deutsche Forschungsgemeinschaft (Sonderforschungsbereich 347: Molekulare Mechanismen unimolekularer Prozesse) is gratefully acknowledged.

\section{References}

[1] M. Maroncelli, J. MacInnis and G.R. Fleming, Science 243 (1989) 1674.

[2] B. Bagchi, Ann. Rev. Phys. Chem. 40 (1989) 115.

[3] H. Heitele, Angew. Chem. Intern. Ed. 32 (1993) 359 and references cited therein.

[4] H. Sumi and R.A. Marcus, J. Chem. Phys. 84 (1986) 4894.

[5] I. Rips and J. Jortner, J. Chem. Phys. 87 (1987) 2090.

[6] B. Bagchi and G.R. Fleming, J. Phys. Chem. 94 (1990) 9.

[7] T.J. Kang, W. Jarzeba and P.F. Barbara, Chem. Phys. 149 (1990) 81 .

[8] G.C. Walker, E. Akesson, A.E. Johnson, N.E. Levinger and P.F. Barbara, J. Phys. Chem. 96 (1992) 3728.

[9] M.J. Weaver and G.E. McManis III, Accounts Chem. Res. 23 (1990) 294.

[10] E. Lippert, W. Lüder and F. Moll, Spectrochim. Acta 10 (1959) 858.

[11] A. Kawski, I. Gryczynski, C. Jung and K.-H. Heckner, Z. Naturforsch. a 32 (1977) 420.

[12] A. Safarzadeh-Amiri, Chem. Phys. Letters 125 (1986) 272.
[13] E. Gilabert, R. Lapouyade and C. Rullière, Chem. Phys. Letters 145 (1988) 262.

[14] E. Gilabert, R. Lapouyade and C. Rullière, Chem. Phys. Letters 185 (1991) 82

[15] W. Rettig, E. Gilabert and C. Rullière, Chem. Phys. Letters 229 (1994) 127.

[16] J. Schroeder, J. Troe and P. Vöhringer, Chem. Phys. Letters 203 (1993) 255.

[17] J. Schroeder, D. Schwarzer, J. Troe and P. Vöhringer, Chem. Phys. Letters 218 (1994) 43.

[18] R. Mohrschladt, J. Schroeder, D. Schwarzer, J. Troe and P. Vöhringer, J. Chem. Phys. 101 (1994) 7566.

[19] J. Schroeder, J. Troe and P. Vöhringer, Z. Physik Chem. 188 (1995) 287.

[20] R. Daum, T. Hansson, R. Nörenberg, D. Schwarzer and J. Schroeder, Chem. Phys. Letters 246 (1995) 607.

[21] T. Hansson, R. Nörenberg, R. Daum, D. Schwarzer and J. Schroeder, Chem. Phys. Letters (1996), in press.

[22] M.A. Kahlow, W. Jarzeba, T.P. DuBruil and P.F. Barbara, Rev. Sci. Instr. 59 (1988) 1098.

[23] K.A. Zachariasse, private communication.

[24] A. Zafarzadeh-Amiri, J. Photochem. Photobiol. A 43 (1988) 43.

[25] M. Maroncelli and G.R. Fleming, J. Chem. Phys. 86 (1987) 6221.

[26] K. Tominaga, G.C. Walker, W. Jarzeba and P.F. Barbara, J. Phys. Chem. 95 (1991) 10475, 10485.

[27] J. Barthel, K. Bachhuber, R. Buchmer, J.B. Gill and M. Kleebauer, Chem. Phys. Letters 167 (1990) 62.

[28] P.V. Kumar and M. Maroncelli, J. Chem. Phys. 103 (1995) 3038.

[29] M. Cho, S.J. Rosenthal, N.F. Scherer, L.D. Ziegler and G.R. Fleming, J. Chem. Phys. 96 (1992) 5033.

[30] P. Vöhringer, D.C. Amett, R.A. Westervelt, M.J. Feldstein and N.F. Scherer, J. Chem. Phys. 102 (1995) 4027

[31] Y. Ooshika, J. Phys. Soc. Japan 9 (1954) 594.

[32] N. Mataga, Bull. Chem. Soc. Japan 36 (1963) 654.

[33] H. Gruen, H. Göorner, Z. Naturforsch. a 38 (1983) 928.

[34] V. Nagarajan, A.M. Brearley, T.J. Kang and P.F. Barbara, J. Chem. Phys. 86 (1987) 3183. 